\title{
Improving Early Childhood Development among Vulnerable Populations: A Pilot Initiative at a Women, Infants, and Children Clinic
}

\author{
Brian A. Ferguson (D), 1,2 Jacy L. Downey, ${ }^{1}$ Amy E. Shriver, ${ }^{3,4}$ Karen L. Goff,, \\ Alyssa M. Ferguson, ${ }^{7}$ and Meena Cabral de Mello ${ }^{8}$ \\ ${ }^{1}$ Des Moines University, Des Moines, IA, USA \\ ${ }^{2}$ University of Louisville Hospital, Louisville, KY, USA \\ ${ }^{3}$ Blank Children's Hospital, Des Moines, IA, USA \\ ${ }^{4}$ Reach Out and Read Iowa, Johnston, IA, USA \\ ${ }^{5}$ Women, Infants \& Children Program, Des Moines, IA, USA \\ ${ }^{6}$ Breastfeeding Coalition of Polk County, Des Moines, IA, USA \\ ${ }^{7}$ UnityPoint Health Iowa, Des Moines, IA, USA \\ ${ }^{8}$ Geneva Foundation for Medical Education and Research, Geneva, Switzerland \\ Correspondence should be addressed to Brian A. Ferguson; batmanferguson@gmail.com
}

Received 24 August 2017; Revised 4 December 2017; Accepted 13 December 2017; Published 15 January 2018

Academic Editor: Olga Capirci

Copyright (C) 2018 Brian A. Ferguson et al. This is an open access article distributed under the Creative Commons Attribution License, which permits unrestricted use, distribution, and reproduction in any medium, provided the original work is properly cited.

Objective. Early childhood development (ECD) programs have demonstrated drastic improvements in survival, growth, health, and social productivity. An ECD pilot intervention was delivered to vulnerable populations of a Women, Infants, and Children (WIC) clinic in Des Moines, Iowa, to assess ECD outcomes and parental receivability in this setting. Study Design. In a randomized, single blinded control initiative, WIC group sessions of mothers (children aged 2 years and younger) were selected for pilot ECD intervention (37 participants) or control (36). Care for Child Development ECD course material was supplemented to intervention groups. Survey results were assessed with paired samples T-testing and by an ANOVA. Results. Pilot session receivability demonstrated significance in all areas relative to control: enjoyment, $p=0.008$; learning capacity, $p=0.011$; and participant sharing, $p=0.023$. Furthermore, the previously validated ECD intervention demonstrated significantly improved cumulative 1 month behavior outcomes following the intervention: $p=0.006$. Conclusions. The WIC setting provides an ideal environment for delivering ECD education beyond traditional counseling in nutrition. High receivability indices among parents demonstrate remarkable capacity for improvement and growth. The significance in receivability, as well as 1 month behavior outcomes, represents parents' overall readiness to enhance the home environment for their child if merely educated how.

\section{Introduction}

The first 1,000 days of life (beginning at conception through two years of age) encompass rapid development, adaptation, and consolidation that takes place in brain structure and function, including peak growth in sensory (seeing/hearing), language/speech, and higher cognitive functions [1-3]. When exposed to home environments that facilitate poor bonding and ineffective levels of stimulation, children at this stage will have significant lifetime developmental detriments $[1,4-6]$.
These detriments include less capacity in education and earnings, poorer health and longevity (especially related to chronic disease), and reduced personal and social adjustment and coping, which results in a greater lifetime stress ratio, withdrawal, anxiety, and aggression $[1,6]$. Exposure to multiple deprivations will synergistically increase these consequences of poor early development [4]. Furthermore, children living among poor communities are at the greatest risk of being deprived during this crucial early period [5]. 
ECD programs within the first 1,000 days have the potential to offset the risk for developmental detriments of vulnerable children, providing better outcomes in terms of health, physical growth, educational attainment, quality of learning, and future societal productivity [7]. Globally, societies that elect to invest in children in the early years-developed or nondeveloped-have the most literate and numerate populations [8]. Not surprisingly, these societies also boast the best health status and simultaneously the lowest levels of health inequality in the world [9]. Moreover, a reduction in inequalities can extend to the next generation as today's children become tomorrow's parents and expose their children to fewer risks, more protective factors, and better opportunities for learning [6]. These ripple effects can extend benefits of investment in ECD over the lifespan of beneficiaries and their families, and in so doing, they are among the most costeffective investments a country can make-supporting both its people and capital gain [10].

When ECD interventions are delayed, the reduction of harm fails to reestablish the original developmental potentials and are much more costly than those within the first 1,000 days $[1,2,9,11-13]$. In this way, trajectories for development follow a general inertia principal-once set in motion, trends are extremely difficult to reverse, which engenders perpetuation of the cyclic trends of intergenerational transmission of poor ECD and poverty $[6,13]$.

Incorporating early child development activities into the health system provides opportunities for reaching vulnerable children that manifest behavior and social issues, poor adaptation, and lower cognitive and educational attainment $[9,14,15]$. An integration of ECD into established maternal and child-health visits ensure cost-effectiveness and time efficiency for both caregiver and health-worker [2,16]. Longterm evaluations in the US have found significant effects of early childhood development interventions that were delivered through the health system and targeted poor and low birth weight children [7, 17-19].

Programs using the WHO and UNICEF early childhood development teaching protocol: Care for Development, have confirmed the ability to produce significant impacts in ECD competencies, as well as intellectual performance, and have demonstrated high perceived acceptance from both providers and participants [15, 20-22].

The Reach Out and Read Program promotes early language development and literacy using the primary health care system. Reach Out and Read has resulted in more children's books in the household, increased reading aloud, and improved language development [23-26].

Among the main reasons for the current lack of investment and public health support in early development is the low level of awareness at the policy and program levels about the critical importance of ECD within the window of opportunity (the first 1,000 days) [2,6]. Additionally, there is a lack of awareness in the role that health services can play in promoting early psychosocial development of children [16], reflecting the need for demonstration projects among vulnerable populations-such as families serviced by the Federal Special Supplemental Nutrition Program for Women, Infants, and Children (WIC).
Currently, there is a push for testing of integrated interventions from the public health fields of nutrition and early childhood development [27]. In previous assessments, both nutritional supplementation and psychosocial stimulation have demonstrated improvements in development, with psychosocial stimulation resulting in improved IQ scores among those that were previously stunted [4]. A review on integration of interventions in the public and primary health setting reveals that integrated community-based strategies for prevention and treatment of malnutrition, along with ECD interventions, have strong evidence for significant benefit and have demonstrated decreased malnutrition mortality (by as much as 55\%) [16]. There is a strong theoretical rationale for integration from both a logistical and financial basis; however, a review of the most recent literature on integration calls for more research into population and nutritional contexts that are most conducive to benefit [27]. There is limited research into the direct integration of early childhood development education into the WIC program; yet, collaboration between existing programs has been shown to enhance delivery of nutritional components [28]. Some challenges to the WIC group setting in the past have been the time consuming nature of prior materials as well as less interactive demonstrations [28]. The Care for Child Development protocol (updated form of the Care for Development protocol for teaching of ECD) includes many previously validated demonstration and group interaction techniques for delivery of ECD materials and would seemingly translate well into the small group WIC setting [15, 20-22, 29, 30].

As the WIC program is primarily focused on nutritional goals, this study demonstrates a pilot ECD initiative, utilizing existing WIC structure, and, while encompassing broader aims, seeks to target improvements in early childhood development practices and examine parental capacity for reception of these materials.

\section{Materials and Methods}

2.1. Participants. The cohort of individuals selected for participation into the study was mothers or fathers with children 2 years of age and less, as identified through regular maternal and child health visits to the urban WIC center located at 2300 Euclid Avenue, Des Moines, IA 50130. Participants were found by existing rosters of preconceived WIC early education groups, which meet biannually and have long been used by WIC to counsel and provide dietary support to new and recently new mothers/fathers. Primary caregivers are encouraged to attend these WIC sessions as they receive their WIC dietary supplement checks following the educational group sessions.

To be eligible for WIC participation, a parent must have a pretax annual household income less than a predetermined local area poverty line adjusted for household size (e.g., for a household of three individuals in Iowa, max total income for inclusion is $\$ 37,296$ (fiscal year 2017)). There was no significant geographic or socioeconomic difference between groups, as all participants were already established as WIC beneficiaries and as such were predetermined (by a healthcare professional) to be parenting a child at "nutritional risk." The 
majority had no greater than a high school education; and the majority of caregivers were mothers (95-97\%).

Participants were asked to participate at the commencement of the WIC group meetings. Recruitment was based on basic understanding of the English language, ability to write for survey completion, and also having a child in the appropriate age range (2 years of age or less).

For the purposes of the study, ECD material was added to existing dietary education curricula for randomly selected groups. Other randomly selected control groups received all survey components; however, no ECD material was supplemented to their usual WIC education. For inclusion there were no age, cultural, or economic constraints for study participation; and all participants were blinded as to which group they were included. Participants could elect to drop the study or decline participation at any time.

2.2. Procedures. All participants were informed and signed consent documents in the presence of WIC staff. If they elected to be involved in the study, they were advised to return in one month's time after the group session to fill out an additional survey. After filling out this secondary survey (hereafter: postintervention survey) they would receive their WIC supplement check. This WIC financial supplement is provided to all WIC involved parents as a baseline public health practice of the organization to assist with the feeding of their child. If they elected to participate in the study intervention, their WIC check was cut to cover one month of support after the first group meeting in order to incentivize a return to the WIC clinic for postintervention survey participation in one month's time. If they returned at one month and completed the postintervention survey to assess home ECD behaviors, they received the remaining portion (2 months) of the financial supplement. If they elected not to participate in the study, they could still participate in the group session as normal; however, they did not receive any ECD intervention survey material and instead received their usual 3-month WIC supplement check. For the regular WIC groups, mothers are additionally incentivized into participation through materials given out during the course sessions to include age appropriate children's books and educational resources. Both the control and intervention groups received the same incentives.

All group sessions took place in the small group meeting room of WIC 2300 Euclid Ave, Des Moines, IA. Group sizes ranged from 1 to 8 mothers (some with accompanying children) with variance due to weather and transportation (a usual and anticipated barrier for these Iowa-based lowincome populations). This intervention sought to generally determine if a pilot ECD intervention would be received well in a group setting at a WIC clinic-as such, groups were treated equally, and we did not seek to measure the impact of group size and its association with ECD material receivability.

For the randomly selected intervention groups, at the initial phase there was $100 \%$ voluntary participation with presentation of the objectives and the structure of the project. Only a single participant was excluded from the ECD surveys by staff due to an inability to complete survey material due to an inadequate understanding of the English language. The total amount recruited for the intervention phase, taking place in January 2015, was 37 participants. At one month's time 26 participants $(70.3 \%)$ returned to complete the postintervention survey. The control group sessions took place over the month of February 2015 and received 36 random participants. The control group was used to compare receivability and parental capacity at the initial session. One-month outcomes from the control group were limited due to high rates of lack of follow-up among the cohort (with 25 individuals not returning). For intervention flow diagram please see Figure 1.

The participants of both the control and the intervention group were blinded. This was a single blinded study as WIC staff were exposed to the teaching material and responsible for directing the group sessions, and therefore they could recognize differences.

2.3. Educational Intervention. The group education material was borrowed from UNICEF's Care for Child Development module. The literature evidence in support of the ECD implementation of UNICEF's Care for Child Development module is established and ideal for group learning settings $[15,20-22,29]$. The complete Care for Child Development module contains activities and learning modules described in the Care for Child Development: Facilitator Notes [30].

For the adaptation of the Care for Child Development module, the facilitator notes section was consulted and the following were borrowed from the model to use for education of parents: an explanation of the significance of ECD, followed by discussions which elicited information about home behaviors relating to early childhood development [30]. Additionally, the following were included: recommendations for play and communication, effective coping mechanisms for stress, and instructions/demonstrations on how to create or use items at home (e.g., toys/puzzles) to shepherd the stimulation of cognitive exercise and ECD advancement [30]. Supplemented to this material was Reach Out and Read supported education about encouraging at home reading practices. Lastly, material was included from the American Academy of Pediatrics' advice on the elimination of television exposure to children less than 2 years of age, and minimal exposure following [31].

The intervention educational session took place during a single one-hour group session and was led by WIC staff. The dynamic of involvement for the participants was discussion engagement through direct questions, with encouragement for concerns, thoughts, and verbal understanding-similar to the discussion dynamic of the regular WIC education groups. To ensure, however, that direction of the discussion was aligned with ECD established principles, interactions were coached through the Care for Child Development counseling protocol to guide recommendations and interactions [30]. These interactions include specifics on greeting the mother in a cordial fashion, making eye contact, encouraging back and forth discussion, using positive verbal and body language, demonstrating play activities as identified in the counseling card, and troubleshooting problems [30]. There was no additional training other than that which was provided through 


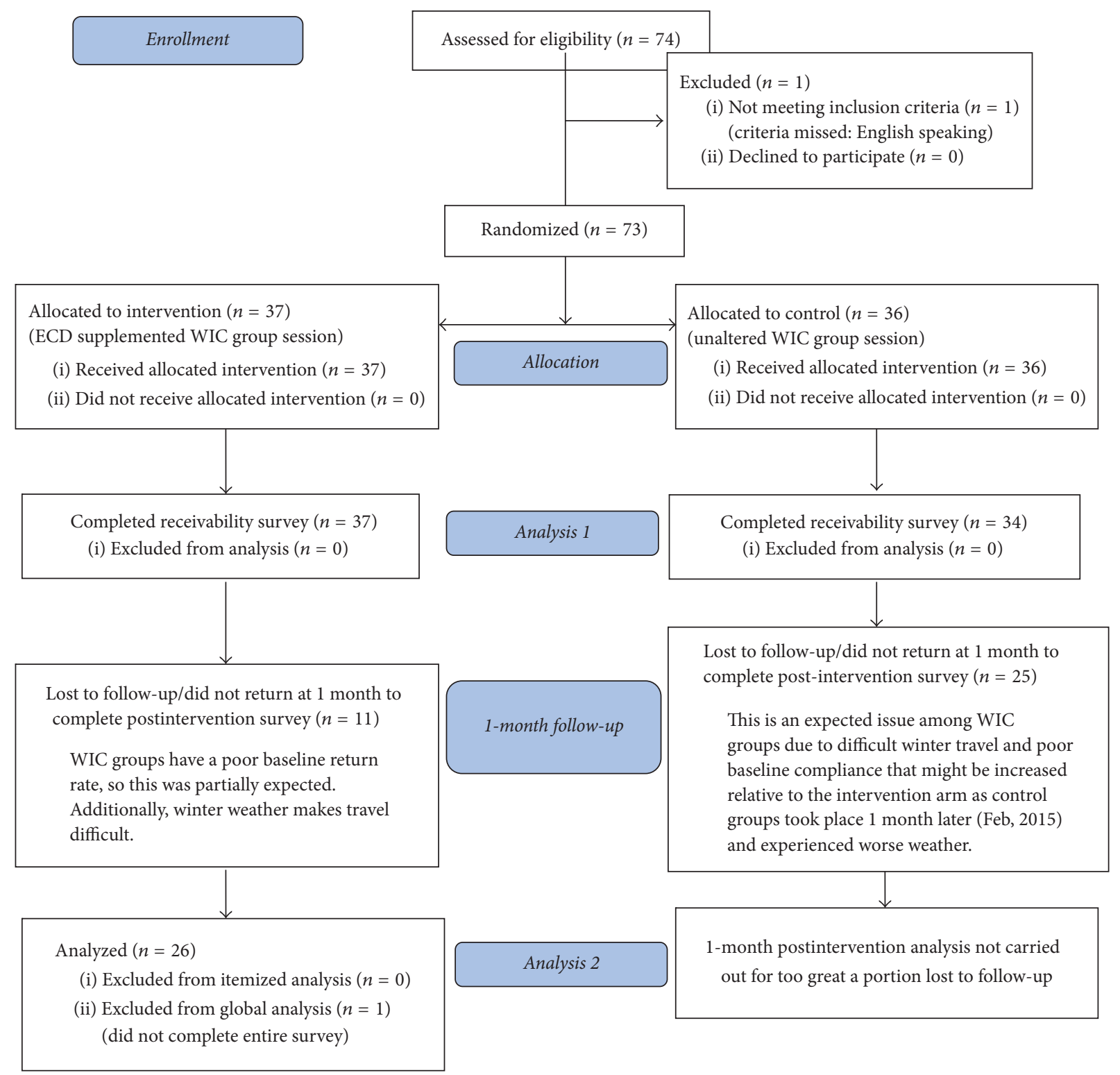

FIGURE 1

reading and understanding the Care for Child Development counseling protocol.

2.4. Survey Collection. ECD behavior surveys were conducted at the initial meeting (preintervention survey) and compared with postintervention surveys completed at one month's time, similar to the timeline of prior ECD intervention assessment of outcomes [15].

The background for the pre- and postintervention ECD surveys of the participants is based on the ECD, "Supportive Environment in the Home" survey, as published in Care for Child Development: Monitoring and Evaluation Guide under, "Tools to evaluate the impact of the intervention" [32]. This survey consists of measures for the home environment to assess pragmatic home ECD practice (e.g., reading aloud, story time, singing songs, exploration, and interactive play).
One hundred percent of participants in both study arms completed this initial survey.

Additionally, a preconceived WIC survey was offered at the end of the teaching session to access agreeability among participants and capacity for learning. One hundred percent of intervention arm participants completed this survey and $94.4 \%$ of control ( 2 participants elected to leave without offering input).

All surveys were completed on paper, created with Microsoft Word software, and were conducted anonymously. Surveys were collected discreetly and remained unopened until the conclusion of the study.

To ensure the methods and surveying procedure were carried out in an ethical and acceptable format, IRB approval was obtained from Des Moines University, and exempt approval was granted. 
TABLE 1: Nested mean scores for supportive early childhood development home behaviors.

\begin{tabular}{lccc}
\hline & Initial $(\mathrm{No})$ & 1 month after $(\mathrm{Ni})^{*}$ & Difference $^{* *}(\mathrm{Ni}-\mathrm{No})$ \\
\hline How often does the mother read to the child & 1.885 & 2.039 & $\mathbf{0 . 1 5 4}$ \\
How often does the father read to the child & 1.308 & 1.269 & -0.039 \\
How often do other family members read to the child & 1.115 & 1.480 & $\mathbf{0 . 3 6 5}$ \\
Decreased television exposure & 1.539 & 2.731 & $\mathbf{0 . 1 9 2}$ \\
How often does the mother play with the child & 2.346 & 1.885 & $\mathbf{0 . 3 4 6}$ \\
How often does the father play with the child & 1.731 & 2.039 & $\mathbf{0 . 1 5 4}$ \\
How often do other family members play with the child & 1.539 & 13.24 & $\mathbf{0 . 5 0 0}$ \\
\hline Total ECD score & 11.68 & $\mathbf{1 . 5 6}$ & \\
\hline
\end{tabular}

*1-month behavior outcomes of the intervention group (participants were matched by completion of pre- and postintervention surveys); ${ }^{* *}$ Ideal early childhood development results are demonstrated by bold differences (1-month outcome mean scores - initial mean scores); ${ }^{* * *}$ Paired sample $T$-test $p$ value $=$ 0.006 .

2.5. Analytic Strategy. Following the completion of survey collection, ordinal variables were transformed into integer values (i.e., each unique answer choice, such as "Few days of the week but not every day," was arbitrarily assigned to an uniquely corresponding integer value, such as " 1 "). Data was then quantitatively analyzed among pre- and postintervention surveys collected from the intervention group. Receivability and parental capacity data for intervention and control were compared with an ANOVA. The data analysis tools were the Statistical Package for the Social Sciences (SPSS) version 22.0, released in 2013, and Microsoft Excel, 2010.

2.5.1. ECD Pre- and Postintervention Survey for Measurement of Home Behavior Change. Ordinal ECD responses consisted of four possibilities: "not at all," "few days of the week but not every day," "one or two times every day," "more than two times, every day". These ordinal responses received a value of "0," "1," "2," and " 3 ," respectively. Integer values assigned to these variables were cumulatively grouped into a final ECD score per participant. The preintervention and postintervention surveys of each participant were nested within either the control or the ECD intervention group. Each postintervention survey was matched to the preintervention survey using the participants' child's name and age, and total ECD scores were compared. All survey data that consisted of a matched pair (pre- and postintervention surveys) was retained. Unfortunately, too many were lost from the control group (with only $30.6 \%$ returning) to accurately assess one-month outcomes of the control participants. However, $70.3 \%$ returned at one month to complete the postintervention survey from the intervention group. Therefore, one-month behavior outcomes were measured from the intervention group and compared with initial surveys.

In order to compare differences before and after the WIC group session, a one-sided paired-samples $T$-test was computed for the intervention arm (based on the hypothesis that ideal ECD behaviors would increase in the home environment following intervention). The null hypothesis was defined to be that no difference is expected between pre- and postintervention total survey scores, with an alpha significance level of 0.05 .
2.5.2. Perceived Receivability Surveys. For the standard WIC receivability surveys completed at the end of the group sessions, ordinal data was transformed to evenly spaced integers (strongly agree $\rightarrow 5$, agree $\rightarrow 4$, okay $\rightarrow 3$, disagree $\rightarrow 2$, and strongly disagree $\rightarrow 1$ ). There were three particular measures assessed: (1) participant elicited enjoyment of the WIC group sessions, (2) participant determined learning amount from the group session, and (3) how much participants felt they were able to share in the group setting. Each of these three measures was averaged by nesting the intervention versus the control. Thereafter, a one-way ANOVA test for a difference among independent means was carried out for each of the three measures.

\section{Results and Discussion}

3.1. ECD Home Behavior. The average ECD one-month behavior outcomes of the intervention are listed in Table 1 . Contained within this table are home behaviors at baseline and changes that took place in the home environment of participants over the one-month interval after the WIC intervention (utilizing data of only the participants that returned for the postintervention survey). The individual outcome scores are group averages for each home behavior (e.g., reading exposure), using ordinal conversions with the following algorithm: (not at all $\rightarrow 0$, few days of the week, but not every day $\rightarrow 1$, one or two times every day $\rightarrow 2$, and three or more times every day $\rightarrow 3$ ). Table 1 also displays average differences for each ECD variable (postintervention outcome score minus preintervention outcome score). Overall, $60 \%$ of the participants in the intervention group improved their global ECD score (excluding a single participant that did not complete the entire postintervention survey), while twenty percent experienced negative growth.

Evident in Table 1, there are profoundly positive home ECD behavior changes (86\%) for the intervention group over the one-month interval-reaching significance when taken cumulatively for a total ECD score (paired sample $T$-test $p$ value $=0.006$ ). This significance found in ECD total score represents an overall ideal behavior change in ECD home practice following the ECD-supplemented WIC intervention. Due to insufficient follow-up from the control group, we cannot decisively state that this observation was independent 
TABLE 2: Comparison of means for teaching session receivability scores.

\begin{tabular}{lccc}
\hline & \multicolumn{2}{c}{ Receivability measures } \\
& I enjoyed WIC group today & I learned something today & I was able to share some things I know with others \\
\hline Intervention & & & $\mathbf{4 . 6 5}$ \\
$\quad$ Mean & $\mathbf{4 . 7 6}$ & 37 & 37 \\
$n$ & 37 & 0.676 & 0.633 \\
$\sigma$ & 0.495 & & $\mathbf{4 . 2 4}$ \\
Control & & $\mathbf{4 . 1 5}$ & 34 \\
Mean & $\mathbf{4 . 3 2}$ & 34 & 0.855 \\
$n$ & 34 & 0.925 & $\mathbf{0 . 0 2 3}$ \\
$\quad \sigma$ & 0.806 & $\mathbf{0 . 0 1 1}$ & \\
ANOVA results $(p$ values $)$ & $\mathbf{0 . 0 0 8}$ & &
\end{tabular}

of expected parental improvement over this one-month interval. However, utilizing the limited one-month outcome data from the control group (11 retained matched pairs who received routine nutrition-only WIC education), 57\% of the competency areas demonstrated equivocal or negative ECD growth. Taken cumulatively, though small, the control group showed no significant changes for the total ECD score (paired sample $T$-test $p$ value $=0.441$ )

3.2. Participant Receivability. The data of the WIC survey which evaluated group session receivability are described in Table 2. The two compared groups listed in Table 2 consist of the control group (no change in preconceived WIC session) and the intervention group (ECD education supplemented to the standard WIC session). All tabulated means are ordinal conversions using the following algorithm: (strongly agree $\rightarrow$ 5 , agree $\rightarrow 4$, okay $\rightarrow 3$, disagree $\rightarrow 2$, and strongly disagree $\rightarrow 1$ ). All three individual markers of a successful WIC group session were higher for the intervention group.

The means of the receivability measures (Table 2) were analyzed with a one-way ANOVA, with the following results: "I enjoyed WIC group today": $p=0.008$; "I learned something today": $p=0.011$; "I was able to share something I know with others": $p=0.023$. Thus, for all three independent variables of the WIC receivability survey there was significance found at an alpha level of 0.05 for the intervention relative to the control. This translates to a higher participant agreeability of the intervention ECD session. This finding also indicates high capacity for learning ECD competencies among WIC mothers. Receivability, as a primary measure of this WIC-based pilot intervention (using previously validated ECD material), suggests that the infrastructure provided by WIC could be an ideal setting for ECD intervention. Therefore, the integration of nutritional and ECD material into WIC groups could represent an ideal strategy to target vulnerable populations utilizing WIC services.

3.3. Limitations. The main limitation of this study is the small $n$ of the selected WIC groups, increasing the chances for a Type I error in reporting significance. Response rates were high in both intervention and control groups in the assessment of receivability; however, logistically the behavior change measure presented follow-up concerns. The intervention study, where significance in home behavior was found, received fairly good secondary response rates (70\%) (relative to the expected winter logistical challenges for vulnerable Iowa-based WIC populations). The control group, which was independently statistically assessed in relation to home behavior change (so as not to bias intervention analytical results), had a much lower response rate at $31 \%$ and was therefore formally excluded from behavior change analysis.

The primary goal of this intervention was the assessment of receivability of ECD materials in the WIC setting, while home behavior change was a secondary evaluation. As such, although home behavior change was observed at one month's time in this pilot intervention, ideally this study would be followed by a longer measure (e.g., 6 months-1 year) with an increased $n$ to assess permanence/long-term sustainability of home ECD behavior changes.

3.4. Recommendations for Future Research. One consistently observed trend within the group discussions was a misunderstanding by parents that certain television programs are acceptable, or even ideal for the development of their infant to two-year-old child. This is contrary to the American Academy of Pediatrics' firm stance that the safe amount of weekly television for children of less than two years is zero hours [31]. Thus an intervention needs to specifically target this topic. The initiative should seek the identification of behavioral change barriers, from where misconceptions concerning television arise, and how to best intervene.

It is also recommended that further research take place on larger scale interventions that incorporate ECD integration with standard WIC nutritional education. Therefore, such studies could develop a national case for broader inclusion of early childhood development practices and education into the already existing WIC infrastructure.

\section{Conclusions}

Relative to the control group, this study has discovered significance in the receivability and parental capacity measures $(p=0.008,0.011,0.023)$. This demonstrates that incorporation of broader early childhood development education into the WIC setting is well received by parents. Concurrently, cumulative one-month behavior outcomes of 
the ECD intervention sessions $(p=0.006)$ are at least optimistic, though limited by comparison data of the control group due to low follow-up.

The informational group sessions were modeled after the proven Care for Child Development intervention; however the time spent on material was terse in comparison to the regular multiday Care for Child Development complete module. These one-month behavior outcomes of this WIC intervention are similar to one-month outcomes using Care for Child Development material delivered through the health care sector [15] and reinforce Care for Child Development's wide application.

Furthermore, the home behaviors that were increased following the intervention, that is, reading aloud, decreased television exposure, and improved play-time (Table 1), are specific behaviors that are part of a more stimulating home environment [15] and carry an established link to better developmental outcomes for children including higher literacy [24]. Therefore, these observed changes are more than just an adoption of arbitrary behaviors-these changes represent areas that optimize the growth of the developing mind of a vulnerable child.

Moreover, as demonstrated by the WIC receivability surveys, parents of vulnerable children both significantly enjoy and believe they are learning from participation in additional ECD education. High receivability indices among these parents of vulnerable children have demonstrated both willingness to learn and high capacity to incorporate key ECD competencies.

This significance observed in receivability of the intervention, coupled with the early integration of ECD principals into home practice (observed by the significance found in pre- and postintervention surveys), represents parents' overall readiness to enhance the home environment for their child if merely educated how.

Although the importance of early childhood development is widely acknowledged, there are significant barriers within the current healthcare environment, including low reimbursement rates, time constraints, and lack of training to conduct these services [33]. However, this study has established that brief and simple early childhood development discussions, even a single encounter, are well received by parents and could be adequate to elicit behavior change at 1 month. Moreover, this study supports that ECD education is not constrained to only the pediatrician's office. Concurrently, this pilot initiative suggests that WIC provides an ideal setting for delivering early childhood development education beyond traditional counseling in nutrition.
Abbreviations
ANOVA: Analysis of variance
ECD: $\quad$ Early childhood development
SEARO: South-East Asia Regional Office
WHO: World Health Organization
WIC: Women, Infants, and Children
UNICEF: United Nations Children's Fund.

\section{Disclosure}

No funding or sponsors supported this publication. All project hours were volunteered by WIC staff and study authors. Brian A. Ferguson wrote the first draft of this manuscript and no grant, honorarium, or other form of payment was given to anyone to produce this manuscript.

\section{Conflicts of Interest}

There are no conflicts of interest among any of the authors of this manuscript, including the study design, collection, analysis and interpretation of data, the writing of the report, or the decision to submit the paper for publication.

\section{Acknowledgments}

WIC staff selflessly donated much time and effort to this intervention, and for that the authors are grateful. The authors are pleased to report that, following this intervention, the WIC staff was able to procure a large children's book donation to distribute universally to all mothers attending WIC groups.

\section{References}

[1] J. Shonkoff and A. Phillips, From Neurons to Neighborhoods: The Science of Child Development, Washington, D.C, USA, 2000.

[2] S. Naudeau, N. Kataoka, A. Valerio, M. Neuman, and L. Elder, Investing in Young Children: An Early Childhood Development Guide for Policy Dialogue and Project Preparation, World Bank, New York, NY, USA, 2011.

[3] G. Doherty, "Zero to Six: the Basis for School Readiness," Applied Research Branch R-97-3E, 1997.

[4] S. P. Walker, S. M. Chang, C. A. Powell, and S. M. GranthamMcGregor, "Effects of early childhood psychosocial stimulation and nutritional supplementation on cognition and education in growth-stunted Jamaican children: prospective cohort study," The Lancet, vol. 366, no. 9499, pp. 1804-1807, 2005.

[5] S. Grantham-McGregor, Y. B. Cheung, S. Cueto, P. Glewwe, L. Richter, and B. Strupp, "Developmental potential in the first 5 years for children in developing countries," The Lancet, vol. 369, no. 9555 , pp. 60-70, 2007.

[6] World Health Organization, Early child development: a powerful equalizer, World Health Organization, Geneva, Switzerland, 2007, WHO Editor.

[7] L. Schweinhart, J. Montie, Z. Xiang, W. Barnett, C. Belfield, and M. Nores, "Lifetime effects: the HighScope Perry Preschool study through age 40," Monographs of the HighScope Educational Research Foundation, vol. 14, 2005, MI: HighScope Press.

[8] J. Heckman and D. Masterov, "The Productivity Argument for Investing in Young Children," National Bureau of Economic Research w13016, 2007.

[9] P. L. Engle, L. C. H. Fernald, H. Alderman et al., "Strategies for reducing inequalities and improving developmental outcomes for young children in low-income and middle-income countries," The Lancet, vol. 378, no. 9799, pp. 1339-1353, 2011.

[10] J. Heckman and P. Carneiro, "Human Capital Policy," National Bureau of Economic Research w9495, University of Chicago, 2003. 
[11] J. J. Heckman, "Skill formation and the economics of investing in disadvantaged children," Science, vol. 312, no. 5782, pp. 19001902, 2006.

[12] P. L. Engle, M. M. Black, J. R. Behrman et al., "Strategies to avoid the loss of developmental potential in more than 200 million children in the developing world," The Lancet, vol. 369, no. 9557, pp. 229-242, 2007.

[13] S. P. Walker, T. D. Wachs, and S. Grantham-Mcgregor, "Inequality in early childhood: risk and protective factors for early child development," The Lancet, vol. 378, no. 9799, pp. 1325-1338, 2011.

[14] WHO/SEARO, UNICEF. Role of the Health Sector in Promoting Early Childhood Development: A strategic framework. SouthEast Asia Regional Office: World Health Organization; 2011, World Health Organization, South-East Asia Regional Office, 2011.

[15] I. O. Ertem, G. Atay, B. E. Bingoler, D. G. Dogan, A. Bayhan, and D. Sarica, "Promoting child development at sick-child visits: a controlled trial," Pediatrics, vol. 118, no. 1, pp. e124-e131, 2006.

[16] Z. A. Bhutta, S. Ali, S. Cousens et al., "Alma-Ata: Rebirth and Revision 6 Interventions to address maternal, newborn, and child survival: what difference can integrated primary health care strategies make?” The Lancet, vol. 372, no. 9642, pp. 972989, 2008.

[17] M. C. McCormick, J. Brooks-Gunn, S. L. Buka et al., "Early intervention in low birth weight premature infants: results at 18 years of age for the infant health and development program," Pediatrics, vol. 117, no. 3, pp. 771-780, 2006.

[18] C. S. Minkovitz, D. Strobino, K. B. Mistry et al., "Healthy steps for young children: sustained results at 5.5 years," Pediatrics, vol. 120, no. 3, pp. e658-e668, 2007.

[19] J. S. Palfrey, P. Hauser-Cram, M. B. Bronson, M. E. Warfield, S. Sirin, and E. Chan, "The Brookline Early Education Project: a 25-year follow-up study of a family-centered early health and development intervention," Pediatrics, vol. 116, no. 1, pp. 144152, 2005.

[20] P. Engle, "The Care for Development Intervention in Kyrgyzstan, Kazakhstan, and Tajikistan," Geneva: UNICEF; 2010.

[21] P. Engle, A. Smeby, and D. Grover, "Care for Development: A process evaluation of ECD promotion in primary health care in 3 countries of Central Asia," Geneva: UNICEF Regional Office CEE/CIS, 2010.

[22] X. Jin, Y. Sun, F. Jiang, J. Ma, C. Morgan, and X. Shen, "Care for Development Intervention in Rural China: a Prospective Follow-up Study," Journal of Developmental Behavioral Pediatrics, vol. 28, no. 3, pp. 213-218, 2007.

[23] B. Zuckerman, "Promoting early literacy in pediatric practice: twenty years of reach out and read," Pediatrics, vol. 124, no. 6, pp. 1660-1665, 2009.

[24] C. C. Weitzman, L. Roy, T. Walls, and R. Tomlin, "More Evidence for Reach Out and Read: a Home-Based Study," Pediatrics, vol. 113, no. 5 I, pp. 1248-1253, 2004.

[25] J. A. Theriot, S. M. Franco, B. A. Sisson, S. C. Metcalf, M. A. Kennedy, and H. S. Bada, "The impact of early literacy guidance on language skills of 3-year-olds," Clinical Pediatrics, vol. 42, no. 2, pp. 165-172, 2003.

[26] C. L. Byington, W. L. Hobson, L. Olson et al., “The good habit of reading (el buen habito de la lectura): parental reactions to an enhanced reach out and read program in a clinic for the underserved," Journal of Health Care for the Poor and Underserved, vol. 19, no. 2, pp. 363-368, 2008.
[27] K. M. Hurley, A. K. Yousafzai, and F. Lopez-Boo, "Early child development and nutrition: a review of the benefits and challenges of implementing integrated interventions," Advances in Nutrition, vol. 7, no. 2, pp. 357-363, 2016.

[28] Y. Greenblatt, S. Gomez, G. Alleman, K. Rico, D. A. McDonald, and M. Hingle, "Optimizing nutrition education in wic: findings from focus groups with arizona clients and staff," Journal of Nutrition Education and Behavior, vol. 48, no. 4, pp. 289-294.el, 2016.

[29] H. Palti, N. Zilber, and S. L. Kark, "A community-orientated early intervention programme integrated in a primary preventive child health service-evaluation of activities and effectiveness," Community Medicine, vol. 4, no. 4, pp. 302-314, 1982.

[30] WHO, UNICEF. Care for Child Development: Facilitator Notes.Geneva: World Health Organization; 2011.

[31] D. A. Gentile, C. Oberg, N. E. Sherwood, M. Story, D. A. Walsh, and M. Hogan, "Well-child visits in the video age: pediatricians and the American Academy of Pediatrics' guidelines for children's media use," Pediatrics, vol. 114, no. 5, pp. 1235-1241, 2004.

[32] WHO, UNICEF Care for Child Development: Monitoring and Evaluation Guide.Geneva: World Health Organization; 2011.

[33] M. Regalado and N. Halfon, "Primary care services promoting optimal child development from birth to age 3 years," JAMA Pediatrics, vol. 155, no. 12, pp. 1311-1322, 2001. 


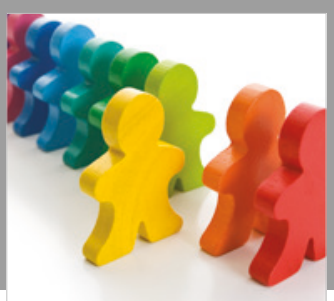

Autism

Research and Treatment
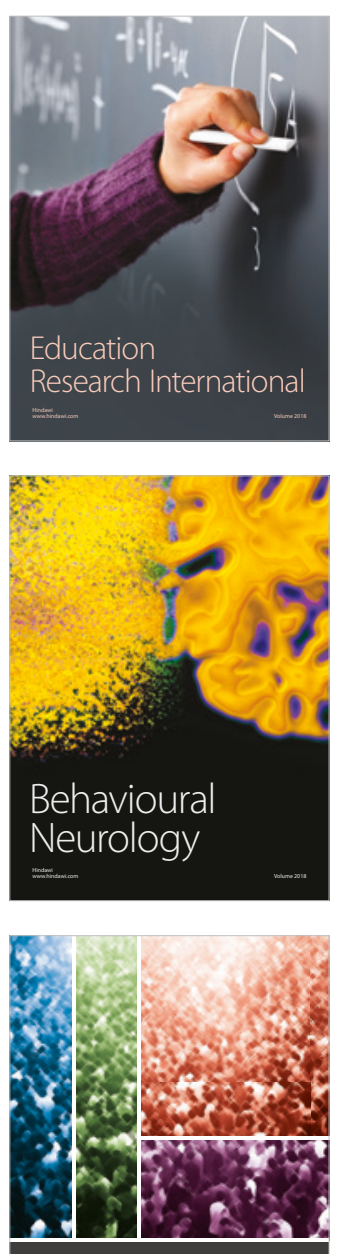

International Journal of

Population Research

$\underline{-m}$

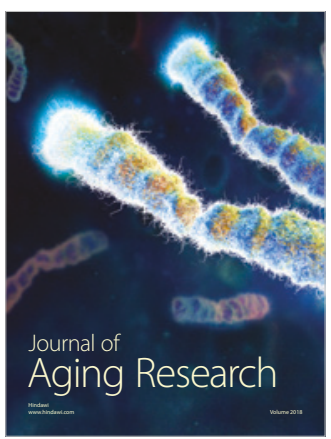

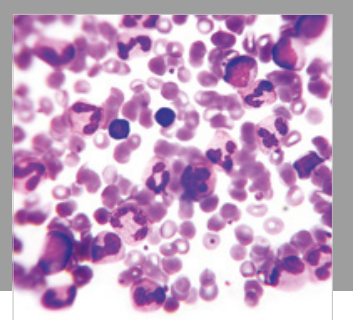

Pathology

Research International$$
=
$$

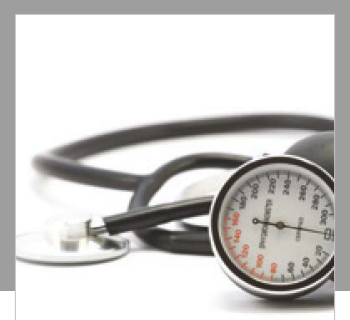

Nursing

Research and Practice

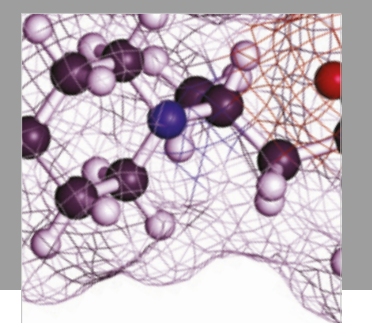

Pain

Research and Management

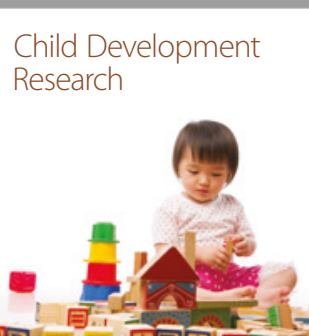

बाD

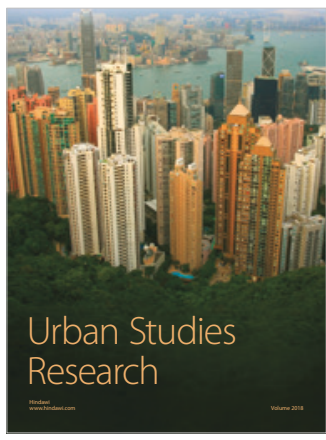

\section{Hindawi}

Submit your manuscripts at

www.hindawi.com
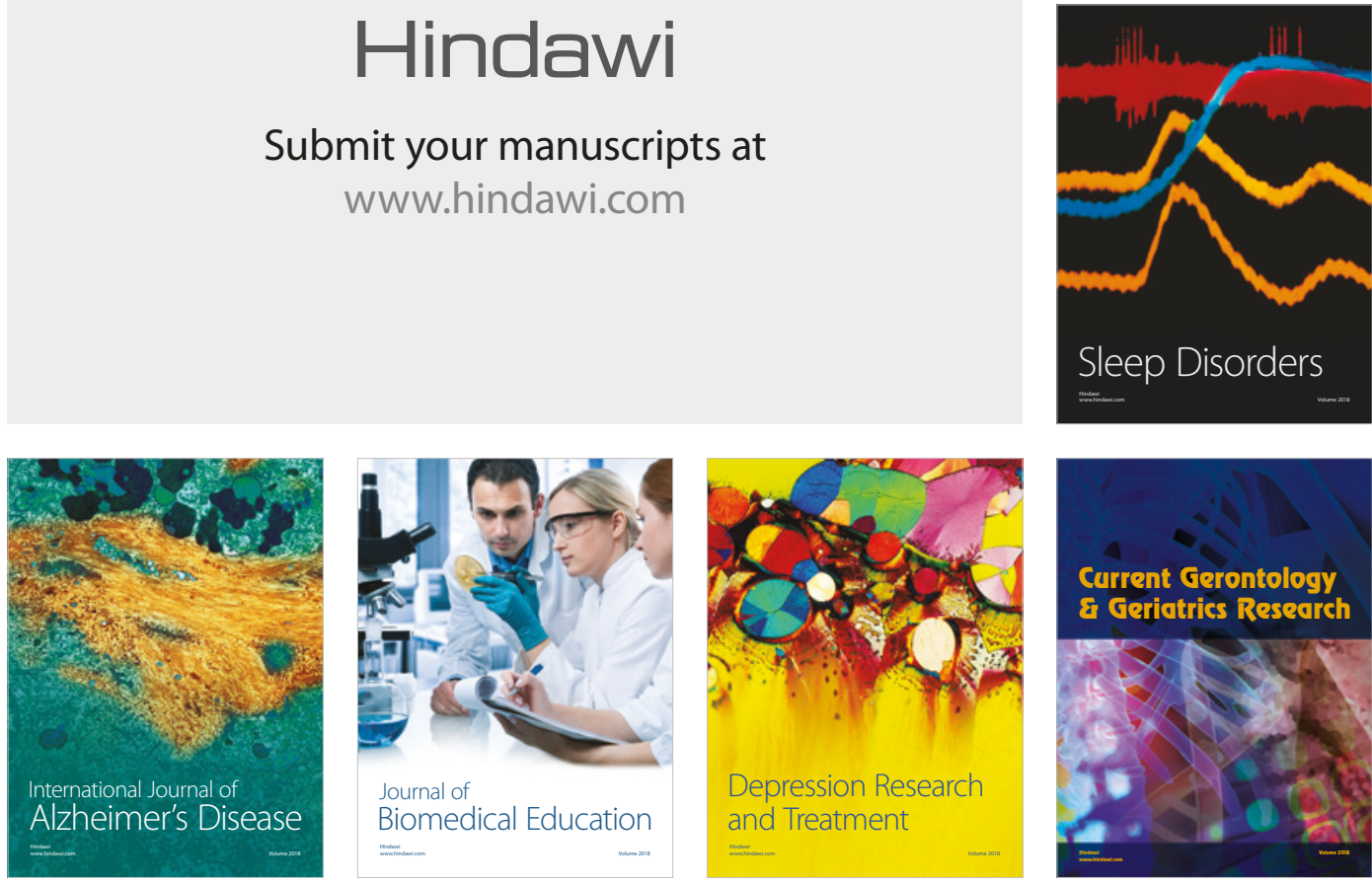

Journal of

Biomedical Education

$=$

smman

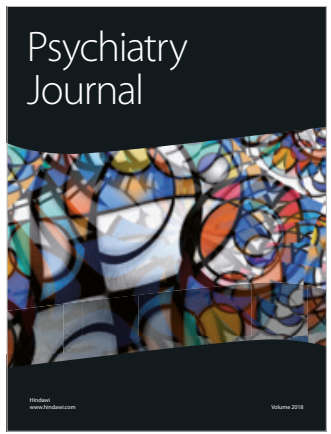

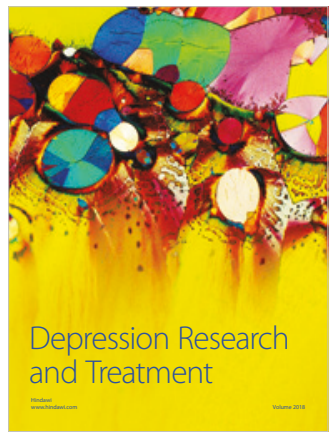
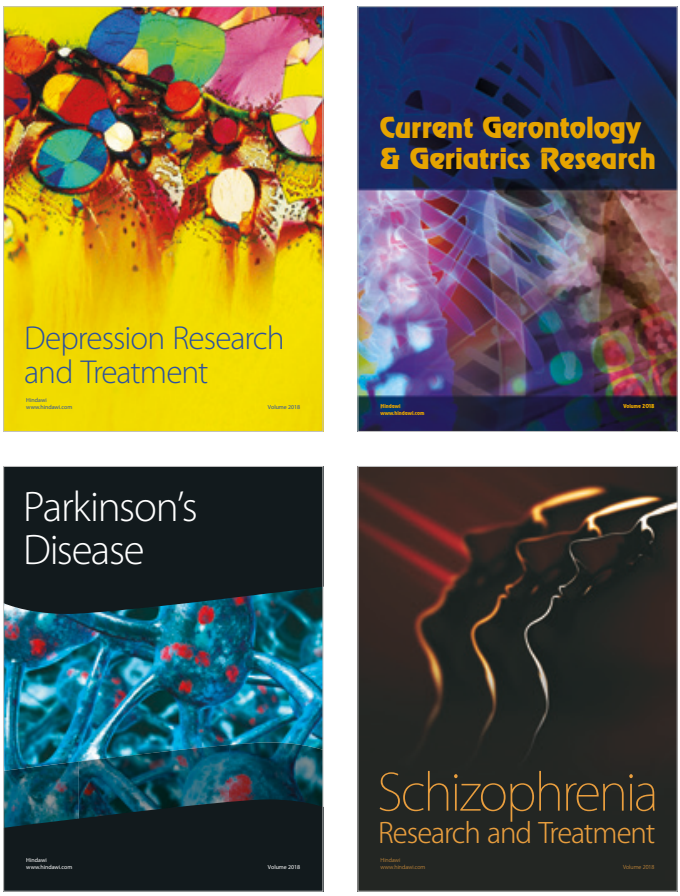